\title{
The collateral history: an overlooked core clinical skill
}

\author{
Donal Fitzpatrick $^{1}$ (1) $\cdot$ Kate Doyle $^{1} \cdot$ Ger Finn ${ }^{1} \cdot$ Paul Gallagher $^{1}$
}

Received: 2 May 2020 / Accepted: 13 July 2020 / Published online: 23 July 2020

(c) European Geriatric Medicine Society 2020

\section{Key summary points}

Aim A comprehensive collateral history is a standard of care recommended both in Ireland, by the Irish National Audit of Dementia Care in Acute Hospitals (INAD) and internationally, by the EuGMS. We sought to establish if this standard was being met.

Findings Only $44 \%$ of patients with cognitive impairment had a collateral history. The level of detail attained was often inadequate and assessment of patients' current and premorbid cognition was poor.

Message Obtaining a comprehensive collateral history is imperative to the diagnosis of delirium and dementia. It is not possible to make a competent clinical assessment or a management plan without exercising this core clinical skill.

\begin{abstract}
Purpose Patients with cognitive impairment are often unable to provide information relating to their pre-morbid cognition and function. Such information is essential to correctly identifying delirium and dementia, as well as making an accurate diagnosis and planning appropriate treatment. It is the standard of care recommended by the EuGMS.

Methods We reviewed medical notes and administered a short questionnaire to nursing staff of a convenience sample of 100 patients aged $\geq 75$ years admitted to medical wards in a tertiary teaching hospital.

Results There were 100 patients with a mean age of 82.3 years, $49 \%$ of whom were female. Of 43 patients with cognitive impairment, 19 (44\%) had a collateral history. Half of the patients described as having dementia did not have any further detail on the severity of dementia documented. Among those for whom a collateral history was obtained, the level of detail regarding pre-morbid cognition, function, mobility, and continence was sparse. Of the total sample, $13 \%$ had formal cognitive testing.

Conclusion Acute illness characteristically causes significant impairments in cognition and function in frail older patients. Identifying and reversing these impairments is impossible without a comprehensive collateral history. It is alarming that such an essential component of clinical assessment is so often disregarded and highlights the lack of awareness from clinicians of the importance of collateral history in the management of patients with dementia and delirium. This must be emphasised in both undergraduate and postgraduate teaching.
\end{abstract}

Keywords Dementia $\cdot$ Delirium $\cdot$ Cognitive impairment $\cdot$ Clinical training $\cdot$ Informant history $\cdot$ Collateral history

\section{Introduction}

The inpatient hospital population is ageing, becoming frailer and cognitive impairment is more prevalent [1]. A collateral history is imperative in diagnosing delirium and dementia [2-5]. Patients with cognitive impairment are often unable to

Donal Fitzpatrick

dfitzpatrick0@gmail.com

1 Geriatric Department, Cork University Hospital, Cork, Ireland provide crucial information relating to their premorbid cognition and function. It is impossible to provide an adequate clinical assessment or plan appropriate management without this knowledge.

A comprehensive collateral history is a standard of care recommended both nationally, by the Irish National Audit of Dementia Care in Acute Hospitals (INAD) [3], and internationally, by the EuGMS [6]. We undertook this audit to establish whether this standard of care was being met for older patients with cognitive impairment admitted to hospital under the medical service. 


\section{Methods}

The audit took place in late 2019 in Cork University Hospital (CUH), a tertiary hospital with 800 beds, in Ireland. It comprised a review of medical notes, the medication prescription record and a short verbal questionnaire delivered to nursing staff.

The sample was a convenience sample of 100 patients over the age of 75 years admitted for longer than $72 \mathrm{~h}$ to a medical ward. Over the course of approximately 6 weeks, we went to each ward in the hospital and included a random selection of five patients from each ward. We repeated this process until we had collected data on 100 patients. Patients admitted to critical care wards, as well as those receiving end of life care, were excluded.

The UK and Irish national dementia audits recommend a collateral history should be taken in all patients with cognitive impairment $[3,4]$. To identify patients who likely had cognitive impairment, and therefore warranted a collateral history, we checked the medical notes to see if the patient had a history of dementia or if a diagnosis of delirium had been made. We checked if delirium screening or other cognitive testing had taken place. We asked the nurse caring for each patient a series of questions, which served as a further measure to identify patients with cognitive impairment (see Table 2). As there is no formal policy of delirium screening in this hospital and some patients likely have an undocumented dementia, we used this questionnaire to increase the proportion of patients with cognitive impairment we identified. However, it is still likely we have underestimated the number of patients with cognitive impairment. We deemed every patient who was confused according to the nursing staff and/or had a documented diagnosis of delirium or dementia as having cognitive impairment necessitating a collateral history.

Among those for whom a collateral history was obtained, the level of detail was assessed in the following domains according to what the doctor had recorded in the medical notes: cognition, function, mobility, continence, details of presenting complaint and living circumstances. Through the questionnaire delivered to nursing staff, we established the patients' current level of function, mobility, continence as well as gaining further information on their cognitive status (see above). Table 1 illustrates how the domains of mobility, function and continence were assessed. Table 2 contains the questions posed to the nursing staff. This study was directed to the collateral history obtained by the medical profession rather than allied health professionals or nursing staff. We examined the collateral history relevant to patients with cognitive impairment. We did not evaluate other reasons necessitating a collateral history, such as transient loss of consciousness.

Statistical analysis was performed using SPSS. All data were anonymised from the time of initial recording. No patient names, record numbers or other unique identifiers were recorded or stored.

\section{Results}

We evaluated 100 patients. The mean age was 82.3 years. The sample comprised 51 males and 49 females. This was a frail cohort of patients with significant levels of impairment of cognition, mobility, and function (see Table 3 for sample characteristics).

A collateral history would have been deemed appropriate because of cognitive impairment in 43 patients out of the

Table 1 Assessment of mobility, function, and continence

\begin{tabular}{|c|c|c|c|c|}
\hline Mobility (degree of assistance required) & Independent & Supervision & Assist of 1 & Assist of 2 or hoist \\
\hline Mobility (mobility aid used) & No aid & Walking stick & Frame or rollator & Immobile \\
\hline $\begin{array}{l}\text { Function (level of assistance required with } \\
\text { personal activities of daily living) }\end{array}$ & Independent & Prompting/supervision & Assist of 1 & Assist of 2 \\
\hline Continence & Continent & Urinary incontinence & Faecal incontinence & $\begin{array}{l}\text { Both urinary and } \\
\text { faecal inconti- } \\
\text { nence }\end{array}$ \\
\hline
\end{tabular}

Table 2 Verbal questionnaire administered to nursing staff
1. As far as you are aware, has this patient been confused since his/her admission to hospital?

2. What level of assistance does the patient require to mobilise?

3. What, if any, mobility aid does this patient use?

4. What level of assistance does this patient require with personal activities of daily living?

5. Is this patient incontinent of urine, bowels, or both?

6. Approximately, what percentage of daytime hours do you estimate this patient spends in bed at present? 
Table 3 Sample characteristics

\begin{tabular}{ll}
\hline General characteristics $(n=100)$ & \\
Mean age & 82.3 years \\
Males: females & $51: 49$ \\
Documented diagnosis of dementia & $18 \%$ \\
Delirium documented & $12 \%$ \\
Confused according to nursing staff $^{\text {Cognitive impairment present }}{ }^{\mathrm{a}}$ & $35 \%$ \\
Nursing home resident $^{\text {Admitted under care of a geriatrician }}$ & $43 \%$ \\
Baseline mobility, continence, and function & b \\
Requiring mobility aid & $5 \%$ \\
Impaired continence & $57.7 \%(n=45 / 78)$ \\
Requiring assistance with pADLs & $24.4 \%(n=10 / 41)$ \\
Current mobility, continence, and function $(n=100)$ & $53.6 \%(n=37 / 69)$ \\
Requiring mobility aid & $78 \%$ \\
Impaired continence & $44 \%$ \\
Requiring assistance with pADLs & $71 \%$ \\
\hline
\end{tabular}

${ }^{\mathrm{a}}$ Cognitive impairment warranting a collateral history was deemed to be warranted in cases where the patient had a documented dementia, a documented delirium and/or where the nurse believed the patient was confused

${ }^{\mathrm{b}}$ The percentages are out of patients for whom a baseline was documented in the medical notes, not the total number of patients

total sample. In 24 of these 43 patients (44\%), a collateral history was not obtained. Of the 19 patients with cognitive impairment who had a collateral history elicited, the history was often incomplete and lacking essential detail. The premorbid level of functional assistance was recorded in $84 \%$ of cases. The baseline mobility aid requirement and level of assistance were documented in $87 \%$ cases. Premorbid cognition was recorded in only $55 \%$ cases. It should be noted that, owing to the study's methodology, we only know if the above domains were documented or not, not the source of the information, i.e. the informant or from elsewhere.

For the patients with cognitive impairment who had a collateral history obtained, the most common informant was the patient's son or daughter (66\%), followed by the spouse $(16 \%)$. Those patients with cognitive impairment under the care of geriatric teams were more likely to have a collateral history obtained. Patients with a documented diagnosis of dementia and with impaired mobility and/or function were more likely to have had a collateral history obtained (see Table 4).

Patients documented as having a diagnosis of dementia comprised $18 \%$ of the sample. Of these, only $61 \%$ had a collateral history obtained. The majority $(56 \%)$ of patients documented as having premorbid cognitive impairment had no detail regarding the nature or severity of the cognitive impairment. Only $13 \%$ of all patients in the total sample $(n=100)$ had formal cognitive testing. Of the 100
Table 4 Associations between variables and obtaining a collateral history in patients with cognitive impairment (Chi square, $n=43$ )

\begin{tabular}{lll}
\hline Variable & $\Phi$ & $P$ value \\
\hline Nursing home resident & 0.145 & 0.348 \\
Admitted because of a fall & 0.082 & 0.717 \\
Dementia (documented) & 0.317 & 0.007 \\
Admitted under geriatric team & 0.294 & 0.013 \\
Assessed by allied health professional & 0.213 & 0.111 \\
Needs assistance with mobility (at time of audit) & 0.376 & 0.001 \\
Needs assistance with pADLs & 0.410 & 0.000 \\
\hline
\end{tabular}

patients, $15 \%$ had a documented delirium, while $38 \%$ were described as "confused" by nursing staff. No patient had a 4AT or equivalent screen completed. Formal cognitive testing was performed in $38 \%$ of the patients described as confused by nursing staff.

Those patients with cognitive impairment warranting a collateral history are frailer. Cognitive impairment was significantly associated with needing assistance and aids for mobilising, needing assistance with pADLs and with incontinence (see Table 5). These patients also had a higher exposure to potentially harmful medications (antipsychotics, opioids, and benzodiazepines).

Regarding nursing home residents, one would expect that much of the information usually acquired through a collateral history would be supplied in transfer documentation from the nursing home. This is not the case, however. The mobility aid required was documented in $80 \%$ of cases. The level of assistance required was omitted in $40 \%$ of cases. Details of premorbid cognition were supplied in $60 \%$ of cases. In those described as having dementia (40\%), no further detail on severity or cognitive scores was supplied. Premorbid levels of assistance with pADLs were recorded in all cases.

Table 5 Associations with cognitive impairment (odds ratios, $n=43$ )

\begin{tabular}{lll}
\hline Variable & OR & $95 \%$ CI \\
\hline Under the care of geriatrician & 1.03 & $0.46-2.31$ \\
Requires assistance with mobilising & 6.37 & $2.16-18.77$ \\
Requires aid to mobilise & 2.90 & $1.02-8.23$ \\
Requires assistance with pADLs & 6.43 & $2.18-19.04$ \\
Incontinent & 8.31 & $3.12-22.13$ \\
Nursing home resident & 4.83 & $0.52-45$ \\
Taking potentially harmful medications $^{\mathrm{a}}$ & 2.31 & $1.02-5.27$ \\
Assessed by allied health professional & 1.81 & $0.77-4.21$ \\
\hline
\end{tabular}

${ }^{a}$ Administered regular antipsychotics, opioids, benzodiazepines 


\section{Discussion}

Obtaining a collateral history is a core clinical skill. This study demonstrates that it is being neglected in everyday practice. In those for whom a collateral history was obtained, the history was frequently incomplete. We found that those with cognitive impairment had higher levels of functional impairment, poorer mobility and were more likely to receive potentially harmful medications.

The task of admitting the patient and acquiring the collateral history generally falls to NCHDs. It is essential that the importance of the collateral history is enshrined in medical education at both the undergraduate and postgraduate doctors. Consultants and other senior doctors must stress the importance of the collateral history to junior colleagues. Nursing staff generally admit patients according to a proforma. This could be replicated for doctors and include a section for collateral history.

There are a variety of screening tools to detect delirium including the 4AT [7] and the CAM (Confusion Assessment Method) [8]. Use of such tools was virtually nonexistent in this hospital. These tools cannot be used properly without some collateral history to establish premorbid cognition. With the help of campaigns such as the UKbased "Think Delirium" [9], there is greater awareness of the importance of recognition and appropriate management of delirium. Delirium has a high mortality and is associated with the onset and progression of dementia [10]. However, it is treatable and once we can identify it there are numerous nursing and medical interventions which can accelerate its resolution and minimise its longterm impact [11].

Similarly, there are numerous efficacious interventions for patients diagnosed with dementia [12]. We suspect there is a large burden of undiagnosed dementia among hospital inpatients, contributed to by neglecting the collateral history. There are several tools to assist in eliciting a collateral history for patients with dementia. These include the IQCODE [13] and the AD8 [14].

Levels of collateral history taking in this audit are very similar to the national standard, recorded in INAD in 2014, where $44 \%$ of patients with known or suspected dementia also had a collateral history recorded [3]. A standardised mental status test was carried out in $43 \%$ of patients in INAD. Similarly, $38 \%$ of patients described as being confused by nursing staff had formal cognitive testing in this study. In the UK national dementia audit, $54 \%$ of patients with dementia had formal cognitive testing [4].

While the collateral history is essential in patients in cognitive impairment, it should not replace or be given more weight than the patient's own account. There is the risk of disempowering a person vulnerable because of cognitive impairment by attributing more value to a caregiver's opinions who may not always have the patient's best interests at heart.

In this study, we focused our attention on doctors-we believe it is a fundamental clinical obligation for a doctor to gather the essential information relating to a patient's illness as well as their premorbid biopsychosocial circumstances. However, the nursing staff also complete a detailed assessment of patients admitted to the ward. In this hospital, the Roper-Logan-Tierney care plan [15] is used. Many of the nurses we spoke to complained about the outdated nature of this proforma. In this model, the nurse documents detailed information relating to a patient's mobility and ADLs. However, it is not clear whether this information is based on the patient's condition at the time of assessment or that prior to their illness. Different nurses have different interpretations. Clearly, both the current and, especially, the premorbid functional and cognitive status should be documented. Doctors, nurses, and allied health professionals should collaborate to ensure that this information is gathered comprehensively but efficiently with appropriate care plans in accordance with the principles of high-quality gerontological nursing.

Despite being some of the frailest individuals in society [16], there is a long history of neglecting the needs of nursing home residents $[17,18]$. The vulnerability and low status of these patients have been unmasked most recently by the disproportionate mortality in nursing homes during the COVID-19 pandemic [19]. The transfer documentation provided when patients are admitted to an acute hospital is inadequate. In particular, the level of detail regarding the patient's premorbid cognition (e.g. severity of dementia, scores on formal cognitive tests) should be documented. Ideally, there should be a universal transfer document containing all pertinent information relating to the patient's medical background, functional and cognitive status, personal preferences, and spiritual beliefs. All this information is included in the Alzheimer Society's personal information document "This is me" [20]. The "This is me" document is useful for all patients living with dementia, not just those in nursing homes. This essential information not only helps in our clinical assessment and care transitions, but also humanises the patients allowing us to relate and empathise with them.

There are several limitations to this study. The sample size is small, consisting of 100 patients. The patients included in this study represented a convenience sample from one hospital. The proportion of patients under the care of geriatric medical teams is disproportionately represented ( $40 \%$ of subjects). The patients were particularly frail with significant premorbid functional and cognitive impairment. Determining when a collateral history would have been warranted is difficult and subjective. We deemed all patients with any degree of cognitive impairment as requiring a collateral history. However, some patients with 
a mild cognitive impairment or mild dementia may not have required a collateral history. Also, because there was no formal delirium/dementia screening in this hospital, we relied on the informal assessment of the nursing staff to identify cognitive impairment in those who did not have formal documentation of impaired cognition. We likely have missed some patients with delirium and/or dementia and therefore we may have underestimated the number of patients with cognitive impairment who should have had a collateral history. It would have been useful to examine frailty scores as a variable; frailty indices are not routinely recorded in this. Despite these shortcomings, we believe the findings are accurate, consistent with our personal experience and likely applicable to similar acute hospitals.

Funding Not funded.

Data availability The datasets are available. The datasets are completely anonymised.

\section{Compliance with ethical standards}

Conflicts of interests None of the authors have any conflict of interest to declare.

Ethics approval The study was reviewed by the Clinical Research Ethics Committee of the Cork Teaching Hospitals and deemed an audit which did not warrant formal ethics review.

Consent to participate The study was deemed an audit by the local ethics committee. None of the patients in the audit were subjected to any intervention. All data collected were recorded with complete anonymity.

Consent for publication All authors have provided consent for publication.

Code availability SPSS proprietary software was used for statistical analysis.

\section{References}

1. Statistics and Analytics Unit; Department of Health; Ireland (2018) Health in Ireland, key trends 2018. Dublin, Ireland

2. Briggs R, O'Neill D (2016) The informant history: a neglected aspect of clinical education and practice. QJM 109:301-302

3. de Siún A, O'Shea E, O'Neill D, McArdle D, Gibbons P, Gilligan MM, Gallagher P (2014) Irish National Audit of Dementia Care in Acute Hospitals: National Audit of Dementia Care. https://www. ucc.ie/en/media/research/irishnationalauditofdementia/INADF ullReportLR.pdf

4. Royal College of Psychiatrists Care Quality Commission (2017) National Audit of Dementia Care in general hospitals 2016-2017: Third round of audit report. London. https://www.rcpsych.ac.uk/ docs/default-source/improving-care/ccqi/national-clinical-audits/ national-audit-of-dementia/round-3/nad-round-3-national-repor t-full-report.pdf?sfvrsn=904926d4_2
5. Dyer AH, Foley T, O'Shea B, Kennelly SP (2018) Cognitive assessment of older adults in general practice: the collateral history. Ir J Med Sci 187:683-687

6. Conroy S, Nickel CH, Jónsdóttir AB et al (2016) The development of a European curriculum in geriatric emergency medicine. Eur Geriatr Med 7:315-321

7. Bellelli G, Morandi A, Davis DHJ et al (2014) Validation of the 4AT, a new instrument for rapid delirium screening: a study in 234 hospitalised older people. Age Ageing 43:496-502

8. Inouye SK, van Dyck CH, Alessi CA, Balkin S, Siegal AP, Horwitz RI (1990) Clarifying confusion: the confusion assessment method. A new method for detection of delirium. Ann Intern Med 113:941-948

9. Healthcare Improvement Scotland; NHS Education for Scotland (NES); Scottish Delirium Association (SDA) (2017) Think delirium, improving the care for older people: delirium toolkit. https ://learn.nes.nhs.scot/2442/rrheal/education-networks/rural-teams -vc-education-network/think-delirium-improvingthe-care-forolder-people-delirium-toolkit.

10. Witlox J, Eurelings LSM, de Jonghe JFM, Kalisvaart KJ, Eikelenboom P, van Gool WA (2010) Delirium in elderly patients and the risk of postdischarge mortality, institutionalization, and dementia. JAMA 304:443

11. National Institute of Clinical Excellence (2019) Delirium: prevention, diagnosis and management Clinical guideline [CG103]. https ://www.nice.org.uk/guidance/cg103

12. National Institute for Health and Care Excellence (2018) Dementia: assessment, management and Dementia: assessment, management and support for people living with dementia support for people living with dementia and their carers and their carers NICE guideline. https://www.nice.org.uk/guidance/ng97/resou rces/dementia-assessment-management-and-support-for-peopl e-living-with-dementia-and-their-carers-pdf-1837760199109

13. Jorm AF, Scott R, Cullen JS, MacKinnon AJ (1991) Performance of the informant questionnaire on cognitive decline in the elderly (IQCODE) as a screening test for dementia. Psychol Med 21:785-790

14. Galvin JE, Roe CM, Powlishta KK, Coats MA, Muich SJ, Grant E, Miller JP, Storandt M, Morris JC (2005) The AD8: a brief informant interview to detect dementia. Neurology 65:559-564

15. Roper N, Logan W, Tierney A (2000) The Roper-Logan-Tierney model of nursing: based on activities of living. Elsevier Health Sciences, Amsterdam

16. Kojima G (2018) Frailty as a predictor of nursing home placement among community-dwelling older adults: a systematic review and metaanalysis. J Geriatr Phys Ther 41:42-48

17. O'Neill D (2018) Reflecting on our perceptions of the worth, status and rewards of working in nursing homes. Age Ageing 47:502-504

18. Bloemen EM, Rosen T, Clark S, Nash D, Mielenz TJ (2015) Trends in reporting of abuse and neglect to long term care ombudsmen: data from the National Ombudsman Reporting System from 2006 to 2013. Geriatr Nurs (Minneap) 36:281-283

19. O’Neill D, Briggs R, Holmerová I, Samuelsson O, Gordon AL, Martin FC (2020) COVID-19 highlights the need for universal adoption of standards of medical care for physicians in nursing homes in Europe. Eur Geriatr Med 1:1-6

20. Alzheimer's Society UK (2010) This is me. https://www.alzhe imers.org.uk/get-support/publications-factsheets/this-is-me

Publisher's Note Springer Nature remains neutral with regard to jurisdictional claims in published maps and institutional affiliations. 